\title{
Spatial spillovers in public expenditure on a municipal level in Spain
}

\author{
Fernando A. López ${ }^{1}$ (D) \\ Pedro J. Martínez-Ortiz ${ }^{2}$. \\ Juan-Gabriel Cegarra-Navarro ${ }^{3}$
}

\begin{abstract}
A key function of local governments is to provide a wide array of public services. The supply of these services has been found to create spatial spillovers among neighbouring municipalities. Although it is generally agreed that spillovers are present in models that explain government expenditures, their type-whether endogenous, exogenous or residual — and sign — whether positive or negative-remain ambiguous. In most cases, a subjective process is used to select the type of spatial regression model used in analysis, with mixed results. Per capita expenditures of ten subprogrammes (Security, Housing, Welfare, Environment, Social services, Employment promotion, Health, Education, Culture and Sport) are analysed for all Spanish municipalities with more than 5000 inhabitants in the 2010-2012 period. A Spatial Seemingly Unrelated Regression methodology in a panel framework is used to incorporate correlation between different subprogrammes and spatial dependence. Our results show that the
\end{abstract}

Electronic supplementary material The online version of this article (doi:10.1007/s00168-016-0780-7) contains supplementary material, which is available to authorized users.

Fernando A. López

fernando.lopez@upct.es

Pedro J. Martínez-Ortiz

pedro.martinezortiz@upct.es

Juan-Gabriel Cegarra-Navarro

juan.cegarra@upct.es

1 Departamento Métodos Cuantitativos e Informáticos, Universidad Politécnica de Cartagena, Cartagena, Spain

2 Grupo de Investigación de Modelización económica y estadística no paramétrica, Universidad Politécnica de Cartagena, Cartagena, Spain

3 Departamento de Economía de la Empresa, Universidad Politécnica de Cartagena, Cartagena, Spain 
three types of spatial effects are present. Furthermore, substantive dependence is positive in most cases, while negative residual dependence is observed in some.

Mathematics Subject Classification C21 · C50 - H72

\section{Introduction}

The last decades have seen a huge increase in research focused on the spatial interactions of public spending policies. The seminal proposals of Case et al. (1993) and Kelejian and Robinson (1993) have led to several research papers that explored the relations between local public spending policies and their spatial spillover effects. At the same time, interest arose in research using models that explain the tax burden of local governments adding spatial effects (e.g. Brett and Pinkse 2000). Both revenue and expenditure policies show spatial spillovers because of the impact of decisions made by authorities in neighbouring regions. For example, a low-tax policy in neighbouring regions can be attractive to both citizens and business, and therefore, the region should have similar tax levels if they do not want citizens to move to neighbouring regions. The same philosophy can be transferred to expenditure in public services, particularly with subprogrammes that are intrinsically linked to citizen welfare. It is evident that certain welfare policies in a small region cannot be blocked for citizens of neighbouring regions. For instance, a low prices policy in the tickets of a local theatre, museum or music festival, due to co-financing by local authorities, will certainly benefit the citizens of neighbouring regions.

These spending policies and their spatial spillovers have generated special interest for investigators. For example, some studies have analysed the total expenditure of local governments (Case et al. 1993; Solé-Ollé 2006) and others analyse specific items: Culture (Lundberg 2006; Werck et al. 2008; St'astná 2009; Benito et al. 2013), Sport and recreational activities (St'astná 2009; Ermini and Santolini 2010), Security (Schaltegger and Zemp 2003), Rescue Services (Hanes 2002) or Environment (St'astná 2009; Ermini and Santolini 2010; Deng et al. 2012; Choumert and Cormier 2011). All these papers propose different models to explain per capita public expending using spatial econometrics models in order to capture spatial spillovers in the regression model (Anselin 1988). The set of data was diverse: in some cases, only the information derived from a single cross section was analysed (Bastida et al. 2013; Ermini and Santolini 2010; Werck et al. 2008; St'astná 2009; Deng et al. 2012), while in other cases, panel data techniques are used (Akai and Suhara 2013; Lundberg 2006; Benito et al. 2013). Moreover, in those papers there is no consensus regarding how to include spatial effects in the model and the specifications vary. It is easy to find specifications that introduce endogenous interaction effects, including substantive spatial dependence, hereinafter spatial lag model (SLM) (Hanes 2002; Ermini and Santolini 2010; Lundberg 2006); or spatial structure in the error term of the regression model, hereinafter spatial error model (SEM) (Bastida et al. 2013; St'astná 2009). Mixed specifications including both types of spatial interactions are also common (Case et al. 1993; Deng et al. 2012). However, the incorporation of exogenous spatial effects is overlooked and this type of effect is not considered in any studies. 
With regards to the sign of the parameters of spatial dependence, the empirical evidence is also ambiguous. In most cases, spatial dependence is positive (Bastida et al. 2013; Revelli 2006; St'astná 2009; Costa et al. 2015), in others it is negative (Hanes 2002; Lundberg 2006; Akai and Suhara 2013) and there are even models that incorporate positive and negative structures with two spatial dependence parameters (Case et al. 1993; St'astná 2009; Deng et al. 2012).

Some of these studies present several methodological shortcomings. One of them is related to the correct selection of the spatial regression models. Some investigators use the prevailing custom of adopting the Florax et al. (2003) methodology based on a comparison of different Lagrange multipliers (LM) tests, but as showed by Mur and Angulo (2009), this strategy is inefficient, leading to a misspecification of the spatial effects. Incorrect model selection of the type of spatial dependence induces bias in parameter estimation and, therefore, an incorrect interpretation of the results. Another is related to the hypothesis of independence between subprogramme expenditures. Some papers estimate the expenditure in several subprogrammes without considering natural relationships (Bastida et al. 2013). Obviously, the hypothesis of independence between subprogramme expenditure is not true and induces inefficient estimation in the model's parameters. Moreover, as highlighted by Case et al. (1993), "there is no reason to assume that patterns of expenditure interdependence are the same for all categories of spending" (p. 303).

Our interest is to provide new evidence while obtaining simultaneous estimations of the expenditure of ten subprogrammes, including spatial effects and correlations between them. We use the Spatial Seemingly Unrelated Regression (SUR) methodology, using model selection based in Mur and Angulo (2009) and extended by López et al. (2014) in SUR. The study uses per capita local Spanish public spending on ten subprogrammes: Security, Housing, Welfare, Environment, Social services, Employment promotion, Health, Education, Culture and Sport.

The paper is structured as follows: the second section contains a brief review of the literature related to possible spatial dependence mechanisms. The third section describes the methodology based on SUR models in a panel data framework. The fourth section presents the most important results and some political implications. In the last section, we present our conclusions, and some limitations.

\section{Spatial effects in public spending}

When local government expenditure responsibilities are decentralized, it is difficult to imagine that local governments make decisions separately. Furthermore, neighbouring municipalities have similar characteristics-demographic, economic and political_as most administrative boundaries are artificial. Therefore, we should expect (dis)similarities in the spending items of spatially close local governments.

\subsection{Sources of spatial dependence}

Three different sources can generate spatial dependence in local expenditure policies:

On the one hand, some spending policies are determined by the specific characteristics of the region (e.g. population structure, taxes and area), which will act as exogenous 
variables in the modelling of public spending and determine specific spending policies. It seems plausible to assume that municipalities that are close to each other have similar socio-demographical and economic characteristics. In this case, spatial dependence would be caused by similar characteristics and citizens' preferences.

Independent variable $X$ of region $\mathrm{A} \rightarrow$ Dependent variable $Y$ of region $B$.

For example, a high percentage of the population over 65 can determine more spending on culture, but nearby municipalities are also certain to have high percentages of population over 65 years and therefore also demand spending in this subprogramme. Therefore, in the model specification, it will probably necessary to incorporate not only the $X$ variables but also some information about neighbouring regions' $X$ variables. This type of spatial dependence may be introduced in the modelling including spatial lag of exogenous factors (WX in spatial econometric terminology). These spatial effects have been overlooked in most of the studies that analyse this topic.

Dependent variable $Y$ of region $\mathrm{A} \leftarrow \rightarrow$ Dependent variable $Y$ of region B.

In this case, from a theoretical point of view, it is possible to differentiate between cooperative and non-cooperative behaviour (St'astná 2009). In the first case, cooperative behaviour emerges from working on joint projects, from sharing experiences and from the learning process. Cooperative mechanisms also arise when local neighbouring governments implement specialization strategies to provide certain services to the citizens (Baicker 2005). In the second case, (dis)similarities can also arise in spending policies as a result of non-cooperative behaviour. Usually, these spillovers emerge as a result of mimetic behaviour by local governments, largely due to the phenomenon known as Yardstick competition (Revelli 2006). Negative spatial spillovers can also arise when there are non-cooperative replacement mechanism strategies. If a local government makes a heavy investment in one item of expenditure, the neighbouring local governments reduce their investment in this item, as their citizens can benefit from the former's services. In order to incorporate those spatial spillovers, the spatial lag of endogenous variables (WY) can be included in the specification.

Finally, local governments located in the same geographic area could be affected by the same external shocks or the same economic policies of higher-level governments, which could also determine the existence of spatial dependence. Moreover, the omission of spatially correlated variables in the specification also contributes to spatial autocorrelation patterns. In those cases, the model can include a spatially autocorrelated error term.

Error term $\varepsilon$ of region $\mathrm{A} \longleftrightarrow \rightarrow$ Error term $\varepsilon$ of region $\mathrm{B}$.

This type of spatial effect has been reported in the case of fiscal policy changes (Allers and Elhorst 2005).

\subsection{Sign of spatial dependence}

The sign of the parameter that captures level of spatial dependence can be interpreted in different ways.

\section{Negative spatial dependence}

Substitutive mechanisms induce negative spatial dependence. For example, the provision of cultural goods or environmental protection in a region can increase the welfare 
of the residents from neighbouring regions. A possible reaction of the local governments would be to profit from these services and decrease their expenditure in these budget items, reallocating resources to other welfare policies. In those cases, we would expect to find models with negative spatial autocorrelation because if a nearby local governments makes a heavy investment in a certain item of expenditure, the neighbouring government will dedicate its resources to other spending policies.

Lundberg (2006) presents empirical evidence of those interaction mechanisms showing the substitutive character of public spending in culture and recreational activities for Swedish local governments. Using SUR methodology (in this paper, it enables correlations for different time periods), he identifies parameters of substantive, negative and significant spatial dependence. In his conclusions, Lundberg maintains that these spending items are used in substitutive form by local governments. Along the same lines, and also with a panel approach, Akai and Suhara (2013) analyse the expenditure on culture for the 1997-2007 period of 45 local governments in Japan. Like Lundberg (2006), the authors identify strong negative spatial dependence using a variety of connectivity matrices.

Another cause that can generate negative spatial dependence structures would be cooperative situations between local governments that agree to design their spending policies establishing specializations. One example is showed by Schaltegger and Zemp (2003), who analyse different spending subprogrammes using panel methodology with spatial dependence in 100 Swiss communes for the 1992-2001 period. The authors identify a strong structure of negative spatial dependence in security expenditures showing cooperative behaviour.

\section{Positive spatial dependence}

Local governments can also emulate their neighbours in decisions regarding their spending policies. There are two cases in which this kind of behaviour is found. The first can be explained by Yardstick competition theory (Revelli 2006), according to which local governments tend to emulate the behaviour of their neighbours trying not to get too far out of line with the policies enacted in nearby jurisdictions as voters make comparisons between the services provided in their setting. St'astná (2009) finds positive spatial dependence in subprogrammes relating to expenditure in housing, regional development, sport and recreational activities. Revelli (2006) explores spatial effects in the provision of social services and finds that the source of positive spatial dependence in social expenditure is a mimetic effect between neighbouring municipalities. Ermini and Santolini (2010) also test the interdependence between 246 Italian municipalities and find evidence of positive and significant interactions both in total expenditure and for different subprogrammes (security, environment, roads). Solé-Ollé (2006) and Bastida et al. (2013), in Spain, also present a model to assess the spillovers resulting from local government policies.

Positive spatial structures can also be found due to cooperation and coordination mechanisms between neighbouring local governments. For example, neighbouring municipalities can work on a set of projects, finance an infrastructure or make investments to protect the environment. There is little evidence of this behaviour in the literature, and we note here the contribution by Reifschneider (2006). 


\section{Positive and negative spatial dependence}

The last situation refers to simultaneous structures of positive and negative spatial dependence. The interpretation in this case is more complex because different mechanisms of interaction are included in the same model. The first paper referring to two simultaneous types of spatial dependence is Case et al. (1993), proposing SARAR model (Mixed regressive-spatial autoregressive model with spatial autoregressive disturbance), identifying substantive $(+)$ and residual $(-)$ spatial dependence. St'astná (2009) also estimates SARAR finding substantive (-) and residual (+) spatial dependence in expenditure in industry, infrastructures and environment protection, whereas in housing and regional development he finds substantive (+) and residual (-) spatial dependence. Gebremariam et al. (2012) estimate a spatial lag with spatial error model and find substantive ( + and non-significant) and residual ( - ) spatial dependence. Deng et al. (2012) in environment protection find substantive (-) and residual (+) spatial dependence in a SARMA model.

\section{Methodology}

\subsection{Spanish institutional framework}

In Spain, there are three vertical government levels: central, regional and municipal. There are seventeen regional governments, the so-called Autonomous Communities or regions (ACs, Nuts II in Eurostat terminology), which have fairly wide-ranging spending responsibilities, mainly the provision of healthcare and education. On the other hand, there are more than eight thousand municipalities $(85.2 \%$ of them are small, with less than five thousand inhabitants). Municipalities have the obligation by law to provide services according to number of inhabitants ( $>5000$, e.g. public parks, market squares, public library; $>20,000$, e.g. social services, sport facilities; $>50,000$, e.g. urban transport). Figure 1 shows the administrative division of Spain without the islands. Municipalities can also provide another set of non-compulsory services (non-compulsory expenditure does not mean it is not essential for citizens). Approximately $25 \%$ of municipal spending is non-compulsory, to ensure equal access to services for all inhabitants. On the other hand, the main sources of municipal revenue are local taxes (two-thirds) and transfers from regional and central government (one-third). With regards to political organization, each municipality has a local government that is democratically elected every four years. This government has total autonomy to decide the municipal budget each year. The budget must be approved by the governing council. Thus, the governing political party has a substantial impact on budget distribution in subprogrammes.

\subsection{Data and variables}

Our dataset includes information about per capita spending in euros, of 1201 Spanish municipalities with a population of more than 5000 inhabitants in 10 subprogrammes for three years, 2010-2012. These municipalities concentrate more than $80 \%$ of the Spanish population. The list of subprogrammes is: Security, Housing, Welfare, Envi- 


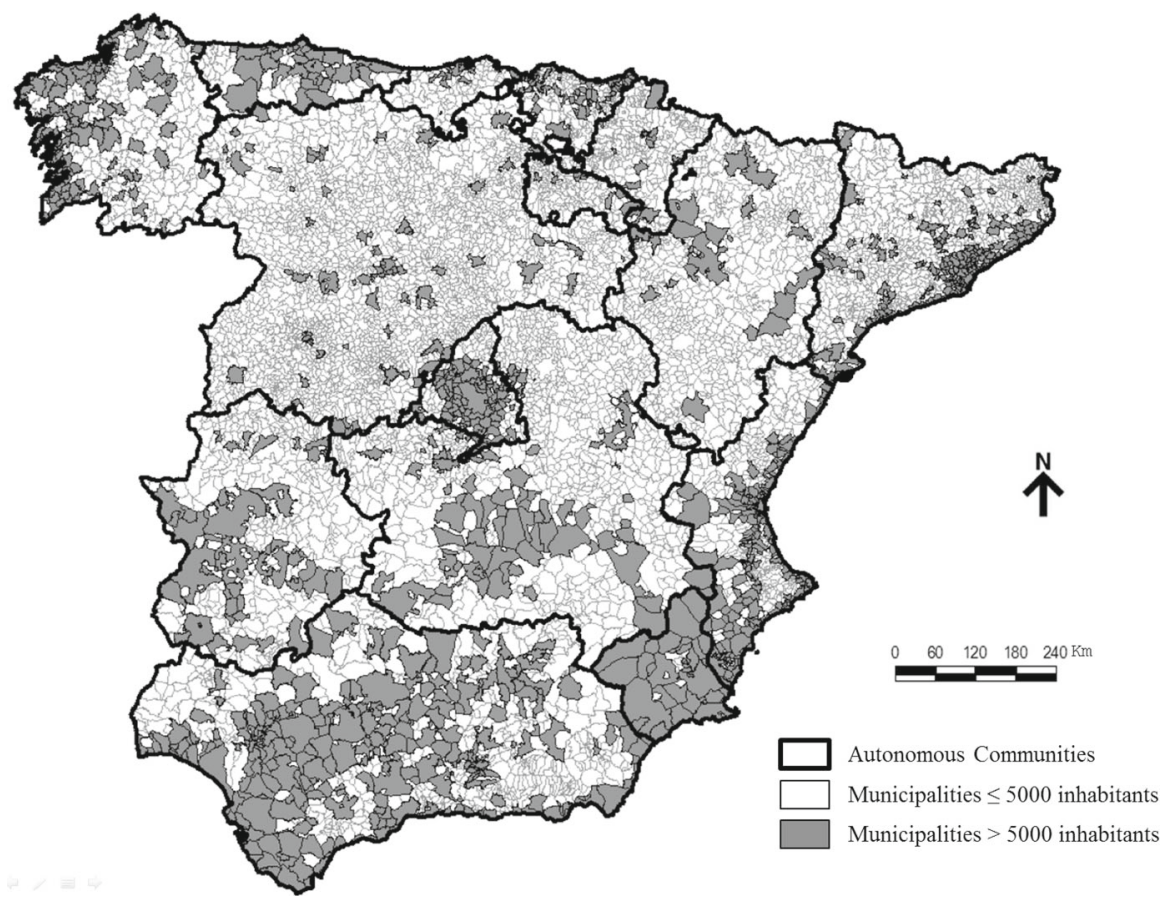

Fig. 1 Administrative division. Autonomous Communities and municipalities

ronment, Social services, Employment promotion, Health, Education, Culture and Sport. Table 1 shows details of budget related to several needs of the population for each subprogramme. The lowest level of spending per capita (mean) is in the Health subprogramme, $8.92 €$ per capita in 2011. This low value is because healthcare is a regional responsibility in Spain, and municipalities only provide some supplementary services. The highest levels of per capita spending (mean) are in welfare with $136.2 €$ in 2011 and housing with $124.43 €$ per capita, associated with local infrastructures (roads, town planning, etc.). These ten subprogrammes cover almost the entire range of local government spending responsibilities, and they represent more than $68 \%$ of total municipal expenditure. The data were obtained from the Spanish Ministry of Finance and Public Administrations.

In addition to these ten dependent variables, there is a series of explanatory factors that have been considered significant in similar studies (e.g. Ermini and Santolini 2010; Benito et al. 2013; St' astná 2009). The set of explanatory expenditure variables along with the source of information and the expected sign are shown in Table 1. Factors have been grouped in four categories: Political factors (Participation; Left-Wing and Alignment), Population structure (Total, Young, Middle-aged and Old), Economic (GDP, Unemployment and Debt) and Geographic factors (Surface and Density). The last columns of Table 1 show descriptive statistics for each analysed year.

The political, demographic and geographic variables are assumed as exogenous factors to explain municipal expenditure. This belief was based on the fact that municipalities have little or no control over demographic variables (such as surface area, 


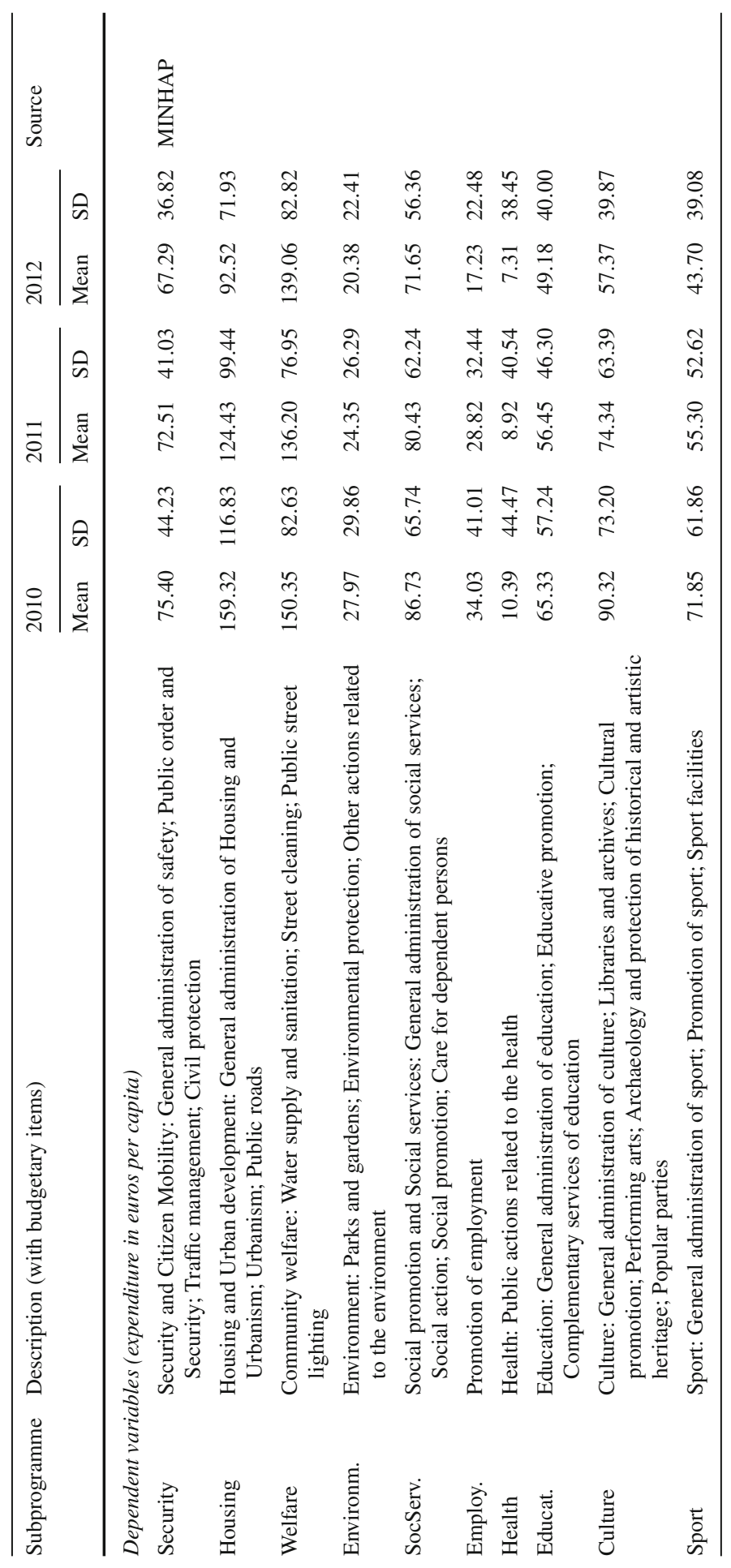




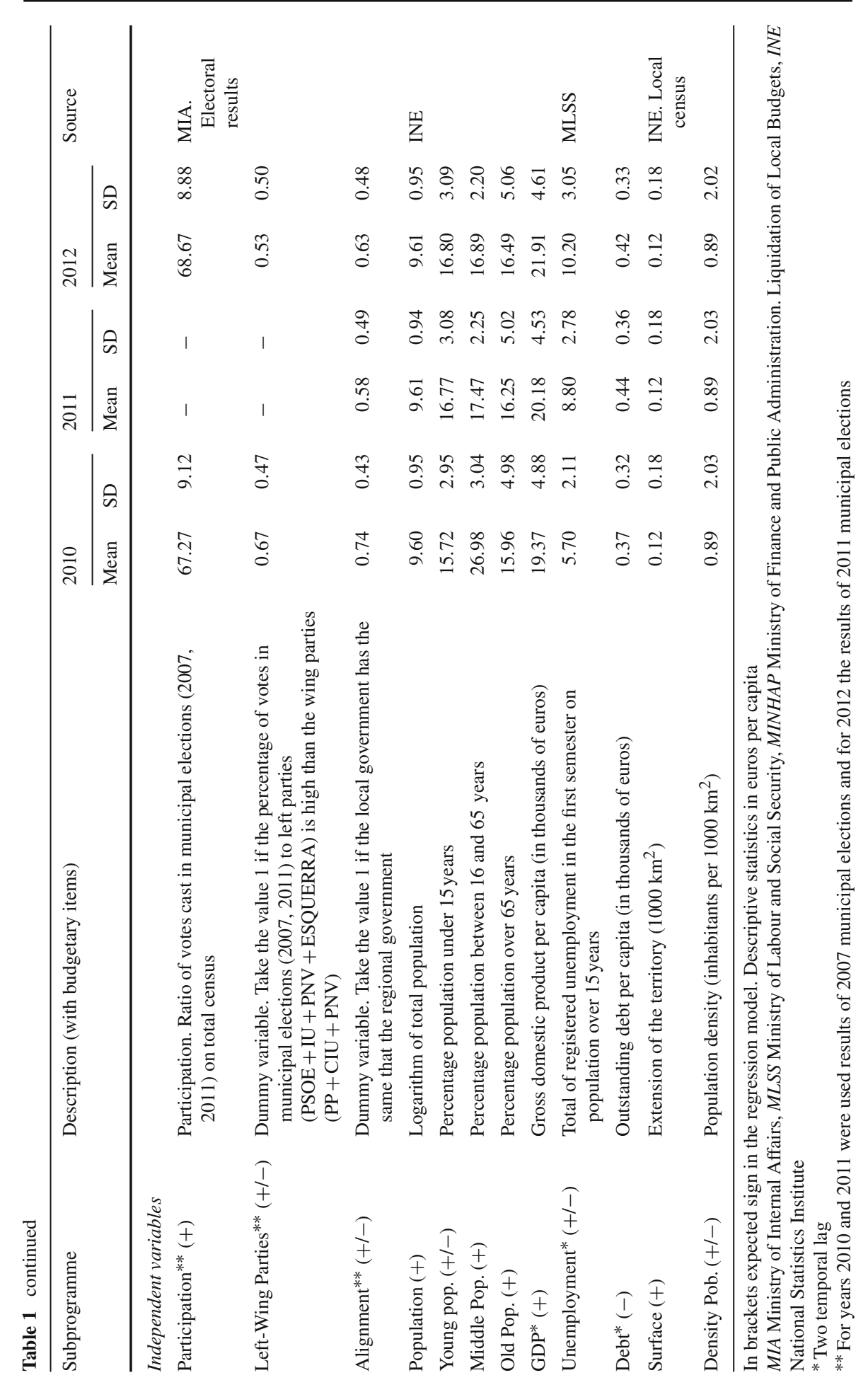


population density and percentage of young people). However, economic factors (GDP, Unemployment and Debt) could be endogenous due to simultaneity. In order to reduce the impact of endogeneity, we consider two time lags as instruments of this variable. Finally, the models include sixteen dummy variables, one for each region in each cross section, in order to control the unobservable spatial fixed effects in the model.

\subsection{Econometric model}

The simplest model, proposed in this study, is a pure SUR model without spatial effects (SUR-SIM):

$$
Y_{\mathrm{gt}}=\mathrm{AC}_{\mathrm{gt}} \delta_{\mathrm{g}}+X_{\mathrm{gt}} \beta_{\mathrm{g}}+\varepsilon_{\mathrm{gt}}
$$

where $Y_{\mathrm{gt}}$ is related to a vector $\mathrm{R} \times 1$ of spending policy $\mathrm{g}(g=1, \ldots, G)$ for period $t$ ( $t=1, \ldots, T$ ) of $R$ local governments. In order to control the impact of the high level of governance due to Autonomous Communities, we include a space-time fixed effect for each region $\mathrm{AC}_{\mathrm{gt}}$ that is a $\mathrm{R} \times 16$ where $\delta_{\mathrm{g}}=\left(\delta_{\mathrm{g} 1}, \ldots, \delta_{\mathrm{g} 16}\right)$ with $\mathrm{AC}_{\mathrm{gt}}(i, j)=1$ if municipality " $i$ " belongs to Autonomous Community " $j$ ". The modelling implies constant $\beta_{\mathrm{g}}$ coefficients in $t$ and enables the inclusion of correlations between residuals of explanatory models of the different spending units, such that $E\left[\varepsilon_{\mathrm{gt}} \varepsilon_{\mathrm{ht}}^{\prime}\right]=\sigma_{\mathrm{gh}} I_{\mathrm{R}}$.

As mentioned in Sect. 2, the theoretical literature assumes the presence of spatial interaction between different local governments. Several models have been proposed (e.g. Brueckner 2003) to incorporate these interactions. The taxonomy of spatial models that we present in this paper is well known (see Elhorst 2014) and we extend in the SUR framework.

The spatial lag of X SUR model (SUR-SLX) is an extension of SUR-SIM including spatial lag of exogenous variables:

$$
Y_{\mathrm{gt}}=\mathrm{AC}_{\mathrm{gt}} \delta_{\mathrm{g}}+X_{\mathrm{gt}} \beta_{\mathrm{g}}+W X_{\mathrm{gt}} \theta_{\mathrm{g}}+\varepsilon_{\mathrm{gt}}
$$

where $W$ is a matrix $\mathrm{R} \times \mathrm{R}$ used to introduce the neighbouring structure (Anselin 1988).

The spatial lag SUR model (SUR-SLM) incorporates a spatial lag of the dependent variable as an explanatory factor.

$$
Y_{\mathrm{gt}}=\lambda_{\mathrm{g}} W Y_{\mathrm{gt}}+\mathrm{AC}_{\mathrm{gt}} \delta_{\mathrm{g}}+X_{\mathrm{gt}} \beta_{\mathrm{g}}+\varepsilon_{\mathrm{gt}}
$$

This specification assumes that spending in a subprogramme $\left(Y_{\mathrm{gt}}\right)$ is partially determined by the weighted average ( $W Y_{\mathrm{gt}}$ ) of spending in neighbouring municipalities. Parameter $\lambda_{\mathrm{g}}$ identifies the intensity and the sign of the impact $(+/-)$ of the policies of neighbouring municipalities.

The classical alternative specification to SUR-SLM is the spatial error SUR model (SUR-SEM) that introduces spatial structure in the residuals of the model and has the following specification:

$$
Y_{\mathrm{gt}}=\mathrm{AC}_{\mathrm{gt}} \delta_{\mathrm{g}}+X_{\mathrm{gt}} \beta_{\mathrm{g}}+u_{\mathrm{gt}} ; u_{\mathrm{gt}}=\rho_{\mathrm{gt}} W u_{\mathrm{gt}}+\varepsilon_{\mathrm{gt}}
$$


These three alternatives can be combined, obtaining several alternatives, the Spatial Durbin SUR model (SUR-SDM) and the Spatial Durbin Error SUR model (SUR$\mathrm{SDE}$ ). Finally, the most general specification is the General Nesting Spatial SUR model (SUR-GNS) that incorporates all types of spatial effects:

$$
Y_{\mathrm{gt}}=\lambda_{\mathrm{g}} W Y_{\mathrm{gt}}+\mathrm{AC}_{\mathrm{gt}} \delta_{\mathrm{g}}+X_{\mathrm{gt}} \beta_{\mathrm{g}}+\mathrm{WX} \mathrm{gt}_{\mathrm{gt}} \theta_{\mathrm{g}}+u_{\mathrm{gt}} ; u_{\mathrm{gt}}=\rho_{\mathrm{gt}} \mathrm{Wu}_{\mathrm{gt}}+\varepsilon_{\mathrm{gt}}
$$

All models are estimated by maximum likelihood. The strategies of selection and comparison between these models are present in López et al. (2014). Finally, unlike in Allers and Elhorst (2011), no total budget constraints are included in this model.

\section{Results}

\subsection{Why a SUR with spatial effects?}

There is high correlation between the ten spending subprogrammes. The upper diagonal of Table 2 shows the correlations between subprogrammes in 2011. For example, correlation between education and culture is 0.27 (0.29 in 2010 and 0.32 in 2012) and correlation between education and sport is 0.17 ( 0.18 in 2010 and 0.22 in 2012). However, there are other subprogrammes that have negative correlations. For example, the correlation between employment and welfare is $-0.08(-0.15$ for 2011 and -0.09 for 2012). These results suggest that there will be similar symptoms in the model's residuals, so SUR methodology should be used (correlations between residuals of final model in lower diagonal of Table 2).

With regards to spatial structure, before testing spatial dependence we have to select a matrix $W=\left(w_{i j}\right)$ that identifies neigbouring municipalities (Anselin 1988). There is no general agreement on how to select this weight matrix although, in our case, the connectivity criterion should assure that the distance between municipalities enables reasonable times for inhabitant flows. In this study, we consider municipalities to be connected if the distance between centroids is less than $\mathrm{d} \mathrm{km}(d=25,30)$. This criterion assigns a large number of neighbours for some municipalities, so we limit the number of neighbours to a maximum of $k(k=8,9)$, selecting the nearest. We consider two different weights $\alpha(\alpha=0,2)$. Formally,

$$
w(\alpha, d, k)_{i j}=\left\{\begin{array}{clc}
d_{i j}^{-\alpha} & \text { if } i \in N_{\mathrm{k}}(j) \text { and } d_{i j}<d \mathrm{~km} \\
0 & \text { in } & \text { other case }
\end{array}\right.
$$

where $N_{\mathrm{k}}(j)$ is the set of k nearest neighbourhoods of municipality $\mathrm{i}$ and $d_{i j}$ is the Euclidean distance. Matrices $\mathrm{W}$ have been standardized by rows as usual. The results of the LM tests of spatial dependence in SUR-SIM model for several $\mathrm{W}(\alpha, d, k)$ are showed in the first rows of Table 3. All LM tests reject the null and confirm a strong structure of spatial correlation in the residual. Given the high values of the LM-SURSARAR tests, it is evident that the baseline model is misspecified in relation to the 


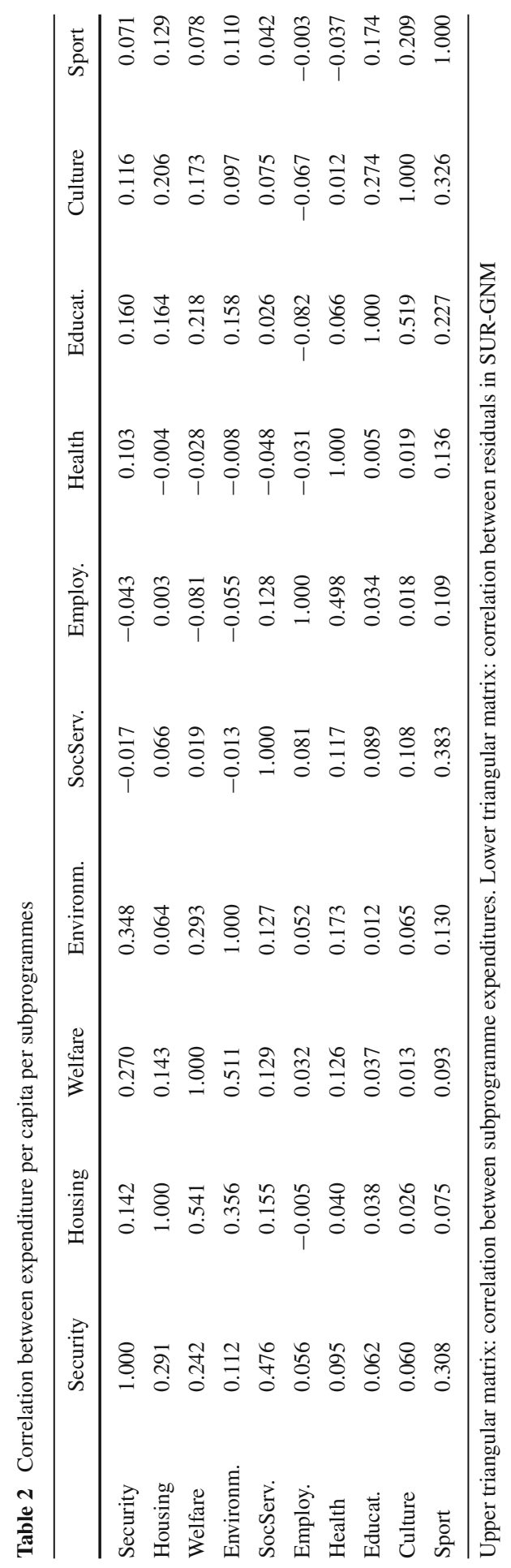


spatial dimension. We decided to select $W=W(0,30,8)$ as the LM-SUR-SARAR takes the higher value though using other parameters in $W$ get similar results. ${ }^{1}$

Finally, alternative methodologies could be used to include spatial effects. Firstly, the spatial simultaneous equation model (e.g. Baltagi and Deng 2015) is a natural candidate but, in our opinion, the dependent variables are not co-dependent. For example, expenditure in the Health subprogramme is not an explanatory variable of expenditure in employment. The dependent variables are simply correlated in their regression error terms. Another alternative could be a dynamic spatial panel methodology with fixed or random effects (e.g. Elhorst 2014, Cap. 4), but those models are more appropriate in case of a single subprogramme and obtain independent estimations. Therefore, a spatial SUR approach is more reasonable for our purpose.

\subsection{Estimation and selection of SUR model with spatial effects}

Several tests are performed to find the most appropriate spatial model. Firstly, the SUR-SLX is estimated to extend the SUR-SIM model including exogenous interaction effects (WX). Table 3 shows the results. The likelihood ratio test is applied ${ }^{2}$ to test the hypotheses of whether spatially lagged independent variables are jointly significant $\left(H_{0}: \theta=0\right.$ in model 2) and the null is rejected ( $\left.L R=445.2\right)$, so SUR-SLX is preferred. In SUR-SLX, all LM tests again showed clear symptoms of spatial autocorrelations in the residuals rejecting the null hypothesis of independence. Now, we consider three alternatives combining different spatial effects: SUR-SDM; SUR-SDE and SUR-GNS. The results for SUR-SDM and SUR-SDE including spatial exogenous effects are showed in the last rows of Table 3. Both models are preferred to SUR-SLX in likelihood ratio terms $\left(\mathrm{LR}_{\mathrm{SLMvsSDM}}=330.6 ; \mathrm{LR}_{\mathrm{SLMvSSDM}}=156.2\right)$, but in both cases, the marginal LM tests ${ }^{3}$ reject the null of independence in the residuals of these models, showing that the correct specification is the model that incorporates all spatial effects, SUR-GNS.

Table 4 shows the results of the final model. SUR-GNS is clearly superior to SURSDM and SUR-SDE in likelihood terms. In this model, the signs of the substantive spatial dependence parameter $\left(\lambda_{\mathrm{g}}\right)$ are positive and significant for eight equations, while residual spatial dependence parameters $\left(\rho_{\mathrm{g}}\right)$ are significant and negative for five subprogrammes. A comparison between the SUR-SLX and SUR-GNS reveals the degree to which the coefficients are over or underestimated. For example, the coefficient of Debt in the Security subprogramme in the SUR-SLX is -1.00 , yet -13.48 in SUR-GNS. Therefore, the impact of Debt is strongly overestimated if spatial effects are not considered. Similar results are obtained for other variables.

\footnotetext{
${ }^{1}$ Similar results with $\alpha=0$ and $d=30 \mathrm{~km}$ and $k=9$ nearest neighbourhoods or $\alpha=0 d=25 \mathrm{~km}$ and $k=8$ nearest neighbourhoods. Not reported to save space but available under request.

${ }^{2}$ For spatial econometric models the use of the standard $R^{2}$ is not appropriate-the $R^{2}$ is uninformative and should be interpreted with caution (Anselin 1988). Therefore, the maximized log-likelihood value is used for models estimated by maximum likelihood as goodness of fit criteria.

3 The marginal test $\operatorname{LM}$-SUR $(\rho \mid \lambda)$ testing for the presence of spatial error structure in a model with spatial lag (SUR-SDM) and LM-SUR $(\lambda \mid \rho)$ testing for the presence of spatial lag in a model with spatial error structure (SUR-SDE).
} 


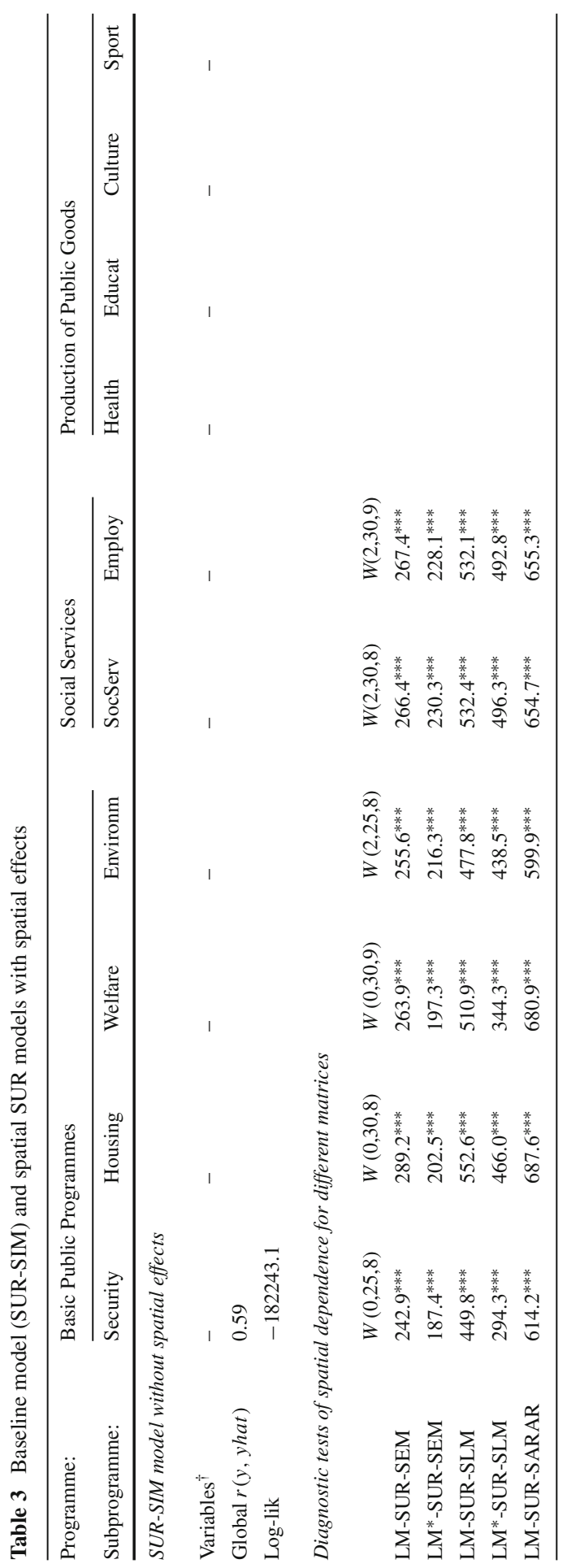




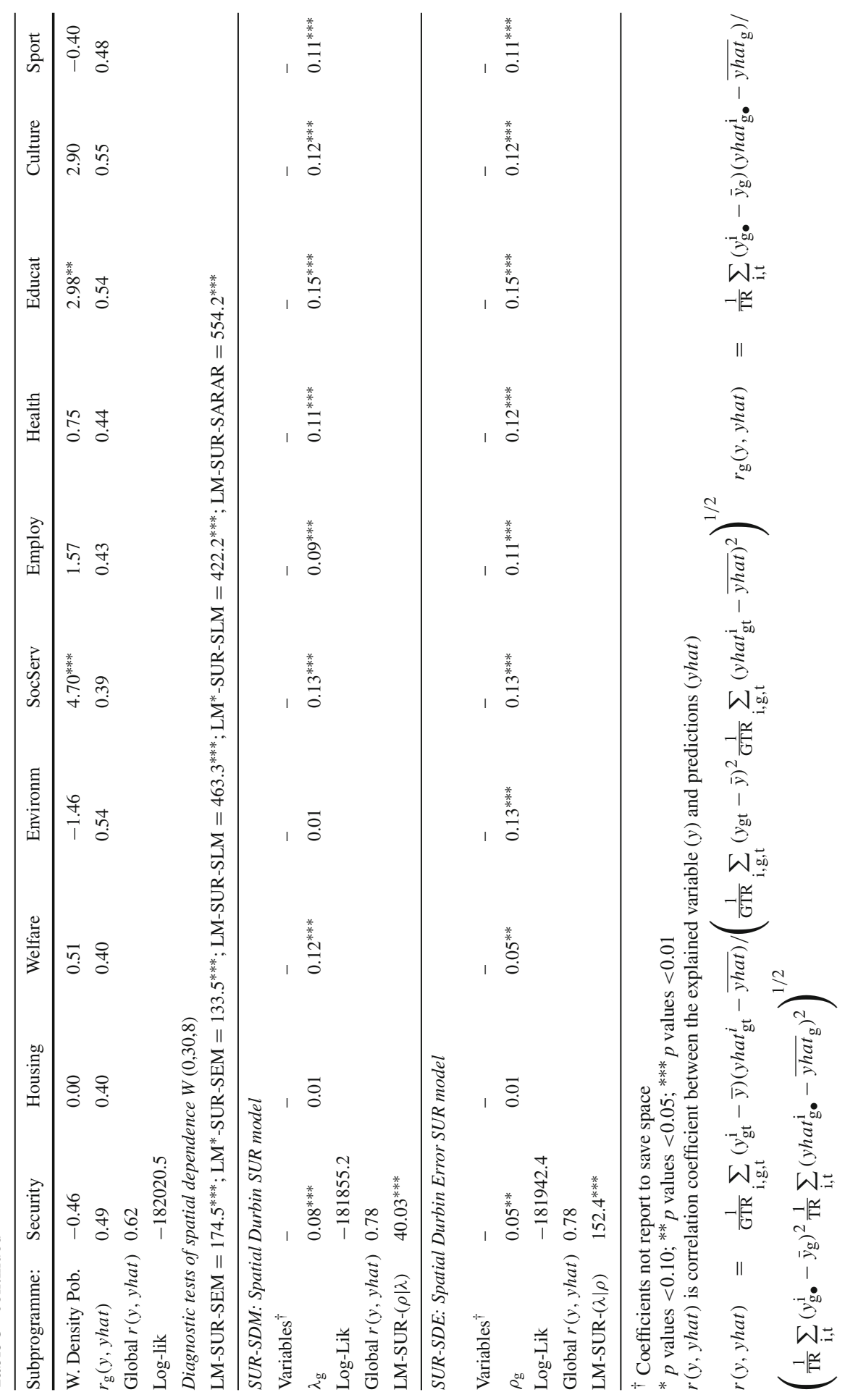




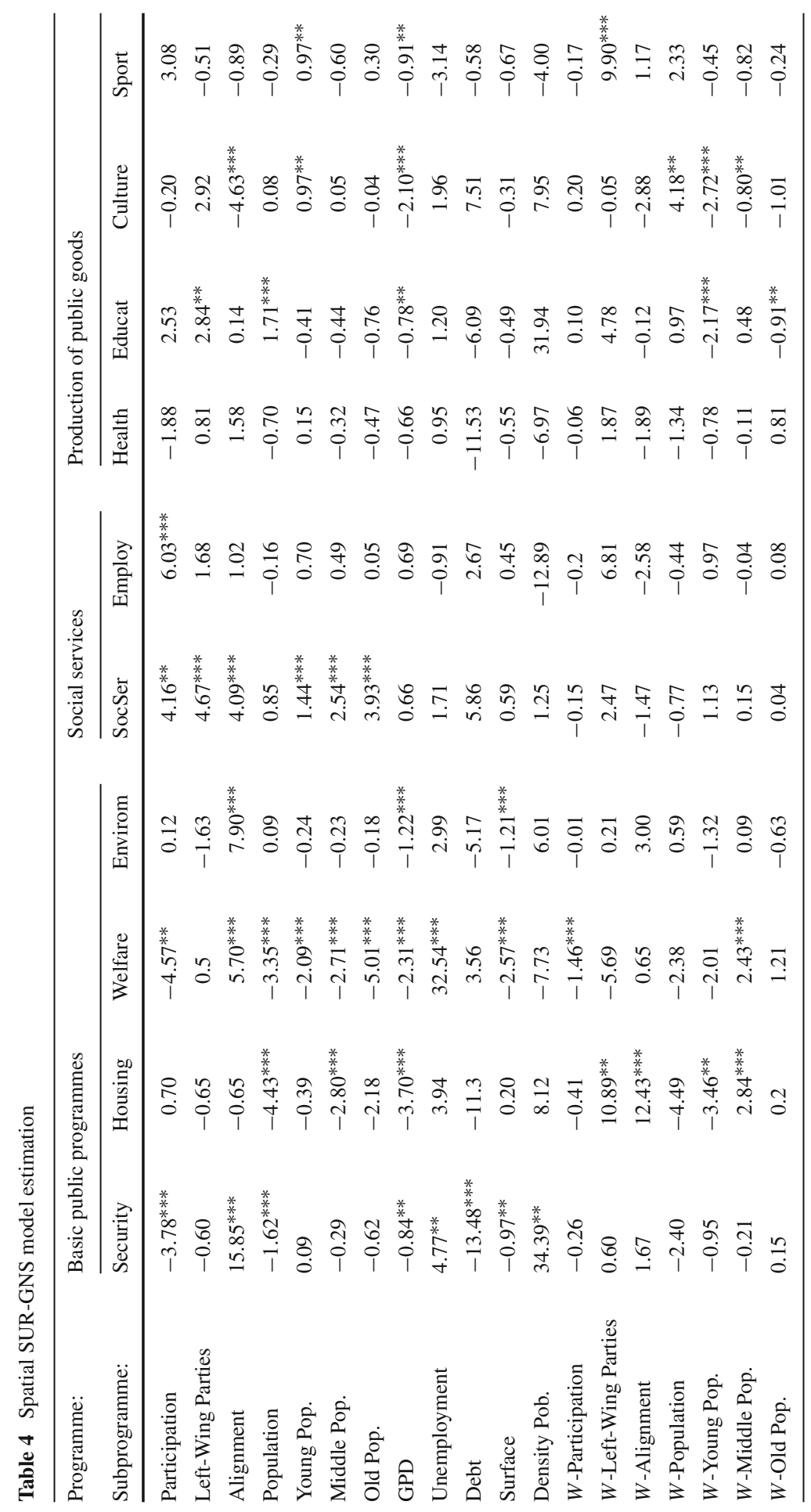




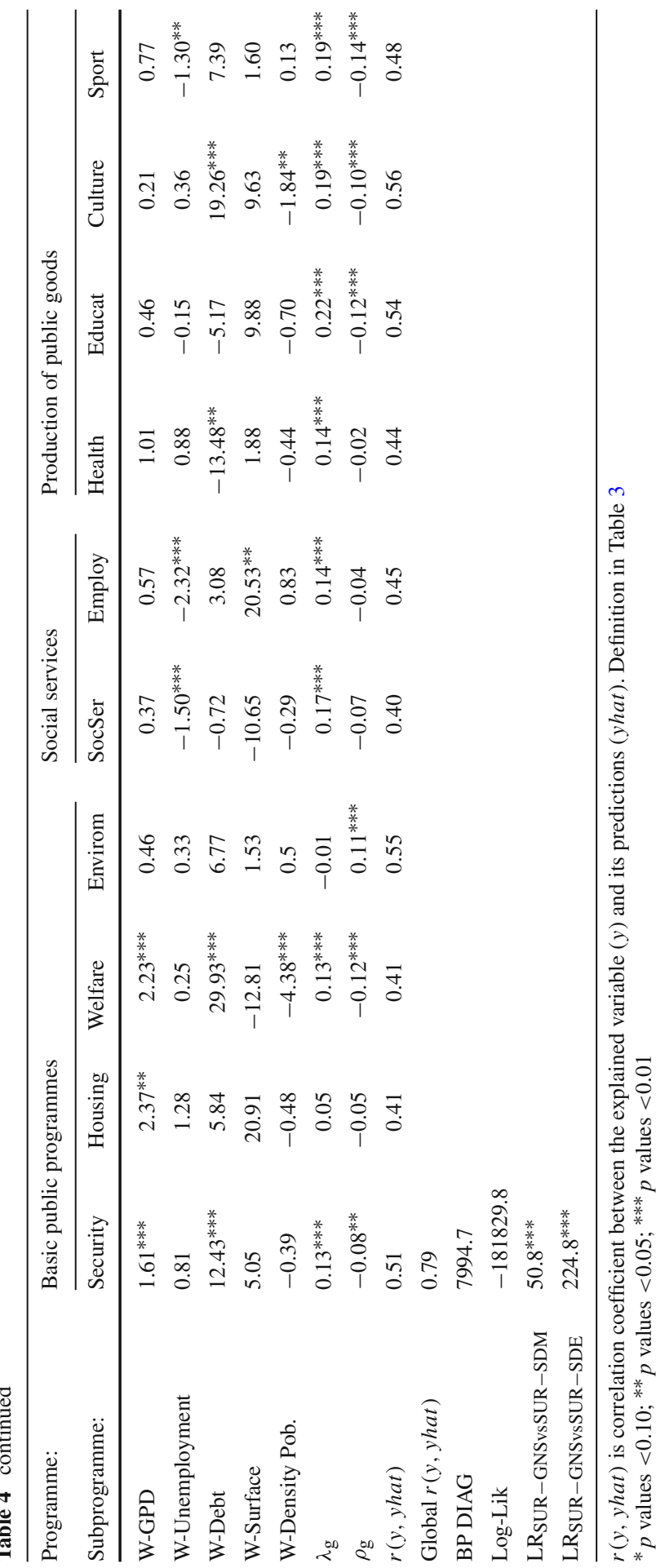




\subsection{Interpretation $\beta_{\mathrm{gk}}$ coefficients in SUR-GNS: direct, indirect and total effects}

For classical regression model estimation, parameter interpretation is easy since parameters can be interpreted as the partial derivatives of the dependent variable relative to the explanatory variable LeSage and Pace (2009). For spatial regression models, parameter interpretation becomes richer and more complicated. A change in the explanatory variable for a single observation can potentially affect the dependent variable of all other observations. In essence, the idea is that spatial dependence expands the information set to include information from neighbouring regions.

The results of LeSage and Pace (2009) can be extended to the SUR framework. Note that

$$
\frac{\partial Y_{\mathrm{g}}}{\partial \mathrm{X}_{\mathrm{k}}}=\left(I_{\mathrm{R}}-\lambda_{\mathrm{g}} \mathrm{W}\right)^{-1}\left(I_{\mathrm{R}} \beta_{\mathrm{gk}}+W \theta_{\mathrm{gk}}\right)
$$

According to Elhorst (2014), these partial derivatives have the following interpretation: if an explanatory variable $\left(X_{\mathrm{k}}\right)$ in a particular municipality changes, not only will government expenditure per capita in that region change, but also government expenditure in other municipalities. Therefore, a change in $X_{\mathrm{k}}$ in a particular municipality has a direct effect on that region, but also an indirect effect on neighbouring municipalities. Note that the ith diagonal element of the matrix of partial derivatives represents the direct effect on the ith region and non-diagonal elements of ith row of the matrix of partial derivatives represent the indirect effects on other municipalities. In order to obtain a global indicator, the direct effect is calculated as the mean of the diagonal elements and captures the average change in government expenditure caused by the change in $X_{\mathrm{k}}$. Likewise, a global indicator of the indirect effects is calculated as the mean of the non-diagonal elements. The total effect is the sum of direct and indirect effects. Finally, note that if $\lambda_{\mathrm{g}}=0$ and $\theta_{\mathrm{gk}}=0$, the indirect effects are 0 and the direct effects are equal to $\beta_{\mathrm{gk}}$.

Table 5 shows the direct, indirect and total effects for the SUR-GNS. The first result to be highlighted is that the exogenous variables have a different total impact for each subprogramme. This result again confirms Case et al. (1993 p. 303). For example, no variable has a significant total effect for the Health subprogramme. This is to be expected because the healthcare system in Spain is the responsibility of the regional government (ACs), and the municipal government only has partial responsibility. However, most exogenous factors have a significant total impact on Security, Welfare, Social services or Culture subprogrammes. These subprogrammes are mainly the responsibility of local government and have a close relationship with council management.

Table 5 provides a great deal of information about the impact of exogenous variables on subprogramme expenditure and requires some comments. Firstly, for political reasons, left-wing parties have a significant total impact on Housing, Social services, Employment, Education and Sport; all the effects are positive, so a higher percentage of left-wing voters increases the total effect on expenditure in these subprogrammes. The left-wing electorate has lower income levels and accordingly supports social policies on wealth redistribution. (Hibbs 1977) and St'astná (2009) show similar results 


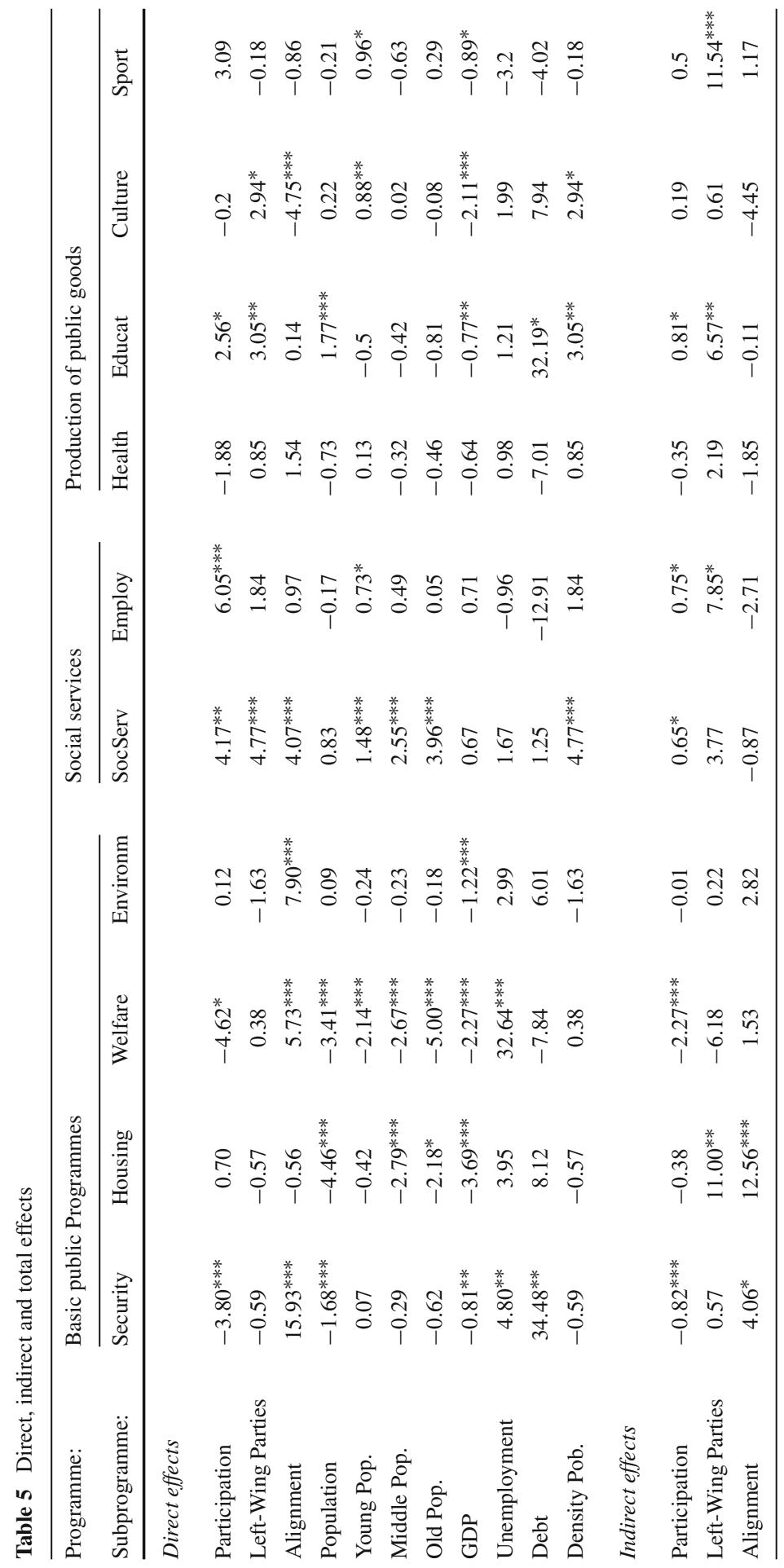




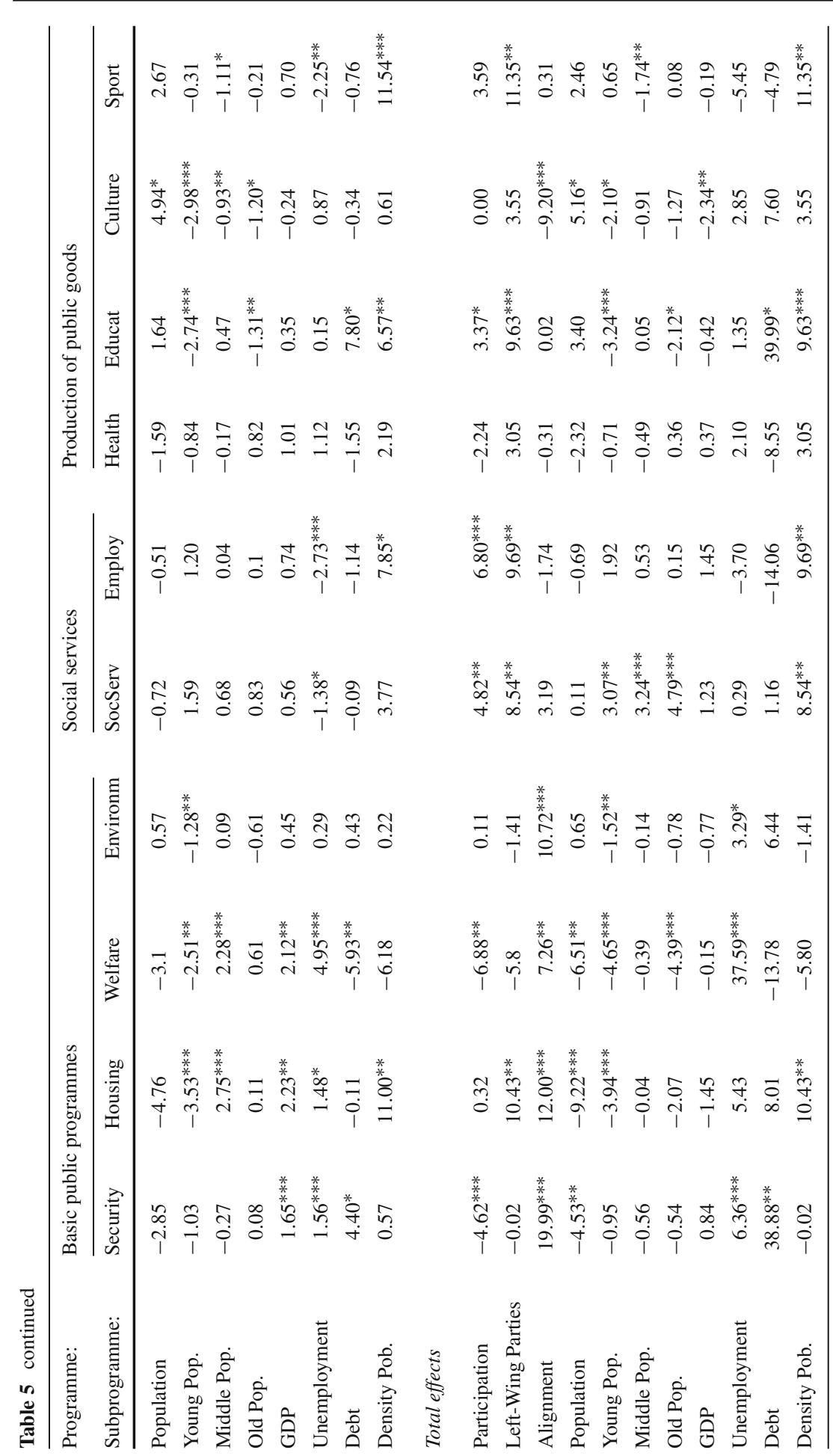


for culture. The empirical results show that these final effects are the results of different processes. For Housing, Culture and Sport, the final effect is only due to indirect effects while, in Social services and Education, the total effect is direct. The participation variable has a significant total effect on Security, Welfare, Social services, Employment and Education. A high percentage of people participating in elections is an indicator of sensitized citizens. High levels of participation increase expenditure in Social services and Education, due to direct and indirect effects. However, the total effect of this variable is negative and significant in Security, Welfare and Education subprogrammes. Finally, alignment variable has a total positive impact on Security, Housing, Welfare, Environment and Culture. It could be due to the competencies transferred from regional governments.

Secondly, demographic factors influence expenditure in several programmes. The total population increases expenditure in Culture due to an indirect effect and reduces spending in Security, Housing and Welfare due to direct effects. The negative sign of those coefficients can be explained as the result of a scale economy process. The young population has a positive total effect in Social Services, while the coefficients are negative in Housing, Welfare, Environment and Education. The percentage of elderly population has a total positive effect on Social services for obvious reasons.

Thirdly, we consider economic determinants. According to GDP, the total effect is significant and negative in Culture. Some authors (Getzner 2004; Lewis and Rushton 2007) present other results and have suggested that higher income levels lead to a greater demand for cultural services. But there is evidence of a negative impact Schulze and Rose (1998) that is not significant (Werck et al. 2008). The debt variable has total positive effects on Security and Education due to indirect effects.

Finally, regarding geographic factors, the effects of the surface area variable are not reported because it has neither a direct nor an indirect impact. Population density is significant and positive in Housing, Social services, Employment, Education and Sport subprogrammes. This result shows that high population density suggests closeness to services and therefore high consumption. Moreover, population density may lead to economies of scale in the provision of local government services, but the empirical evaluation of the impact of this variable shows mixed results. By example, a similar result was found by St'astná (2009) and opposite by Bastida et al. (2013).

\subsection{Spatial spillovers in public expenditure on a municipal level}

At this point, it is of interest to provide an interpretation of the spatial dependence coefficients. Firstly, exogenous spatial lags are significant in most subprogrammes. For example, five exogenous spatial lags are significant in Housing, Welfare and Culture. None, however, are significant in Environment. These results confirm the importance of this type of spatial dependence that has been overlooked in studies of spatial spillovers in municipal spending. Secondly, it is evident that an increase in a municipality's expenditure has an impact on the spending of neighbouring municipalities because endogenous spatial interaction effects are present in local government expenditure. For most subprogrammes, the $\lambda_{\mathrm{g}}$ coefficients are significant and positive (not significant in Environment but $\rho_{\mathrm{g}}$ is positive and significant). Substantive spatial dependence levels 
are similar for the eight subprogrammes with significant coefficients, ranging from 0.13 to 0.22. Case et al. (1993), Baicker (2005) and Bastida et al. (2013) obtained similar spatial dependence values. This type of positive spatial dependence reflects how a municipality influences variations in neighbouring municipality budgets, and has been associated to a comparison, yardstick or coordination mechanism (Brueckner 2003).

Thirdly, there is a negative spatial dependence structure in the residual in five subprogrammes. The values of $\rho_{\mathrm{g}}$ are negative and significant for three subprogrammes included in "Production of Public Goods" (Education, Culture and Sport), and in the Security and Welfare subprogrammes. Case et al. (1993) obtained exactly the same result (positive and negative spatial dependence in the same model), and the authors attribute it to a positive effect of substantive spatial dependence that is accompanied by a nonlinear structure in the residuals (negative). The study by Revelli (2001, p. 1101) attributes the simultaneous presence of the two forms of spatial dependence to the effect of a multi-level government structure. This could be the situation in Spain where the three different administrations share spending policies. But this multi-level effect should appear in the model with positive signs both in substantive and residual spatial dependence due to expenditure policies in a region (ACs) and would be the same in every municipality. Moreover, the SUR-GNM model included sixteen dummies to control fixed spatial effects (not reported to save space), so that is probably not the cause in Spain.

In general, the spatial econometric literature mostly shows models with positive spatial dependence, but there is evidence that in those models there is always some level of hidden negative spatial autocorrelation (Griffith 2006). Following the idea of Griffith (2006) and Griffith and Arbia (2010), spatial autocorrelation is always a mixture of positive and negative spatial autocorrelation, but negative spatial autocorrelation is only detected at a fine geographical resolution since aggregating areal units into a coarser resolution will average dissimilitude. But this is not the case for our results because in the sample all municipalities are medium sized or large (>5000 inhabitants). On the other hand, the spatial econometric literature relates spatial correlated error terms to the subspecification of the model, where some exogenous variables are missing and are themselves spatially dependent (Brueckner 2003, p. 184). In this respect, a possible cause of alternation in the signs of spatial dependence could be attributed to the fact that local governments make decisions about municipal spending based on two criteria. They first imitate what occurs in neighbouring municipalities (positive and substantive spatial dependence) because nearby municipalities have the same political sign. On the other hand, local governments adopt political decisions hardly explained using exogenous factors in an econometric model and strategically planning municipal spending through substitutive mechanisms following the idea proposed by Lundberg (2006). These substitutive mechanisms induce a shock in the model, usually related to residual spatial dependence (negative spatial error dependence). Note that the negative sign of the spatial dependence in the error term is present largely in the four "Production of Public Goods" subprogrammes and Welfare, which are more likely to show flows of citizens who benefit from the services provided by neighbouring municipalities. Those results confirm, and extend to several subprogrammes, the hypothesis presented by Lundberg (2006). 


\subsection{Policy implication}

Municipal managers are that most of the services that they provide are also enjoyed by inhabitants from surrounding municipalities, and vice versa. The empirical evidence shown in this study reveals the important impact of spatial factors on determination of spending in most of the analysed subprogrammes. Note that in our modelling, we went from a correlation between observed values and predictions in the baseline model of $r(y, y$ hat $)=0.59$ to $r(y, y h a t)=0.79$ adding only spatial factors. This result shows effective interaction between local communities.

Several countries have pursued inter-municipal co-operation as an instrument for local government reform. Spain is not alien to this question and debate about the rationalization of local administration grows in periods of economic crisis, when resources are limited. Our empirical findings confirm that, in practice, municipal managers take into account the demographic, economic and social structure of the surrounding municipalities and do not develop isolated policies. Spain is a mosaic of more than 8000 municipalities, most of them very small, and debate about the fusion of local municipalities is alive in Spanish economic circles. The results showed in this paper confirm that the fusion of municipalities in Spain would have a small impact in terms of municipal spending. Although our findings are based on municipalities larger than 5000 inhabitants, we are confident that similar results would be found for smaller municipalities.

The development of this kind of model can also be a useful tool for identifying the main determining factors of municipal spending and to elaborate equitable laws on the reform of state funding for local communities. The empirical approach adopted in this paper can be replicated in institutional settings other than Spain one to increase understanding of the consequences of local council partnerships on fiscal policies at lower levels of government.

\section{Conclusions}

Researchers have shown evidence that confirms the hypothesis that local authorities do not make budgetary distribution decisions in isolation. On the contrary, they consider the behaviour of nearby local governments. This hypothesis has already been tested in empirical studies for several countries (e.g. Werck et al. 2008 in Belgium; Lundberg 2006 in Sweden; St' astná 2009 in Czech Republic; Ermini and Santolini 2010 in Italy; Allers and Elhorst 2011 in Netherlands; Costa et al. 2015 in Portugal), but to date, there is little literature related to Spain (Bastida et al. 2013; Solé-Ollé 2006).

Our results shown a correlation structure between the residuals of the model, which supports the SUR estimation while confirming the presence of a strong spatial structure. The results show the presence of three different sources of spatial effects: endogenous, exogenous and residual. Firstly, the exogenous spatial factors show that local governments are influenced by the characteristics of their neighbours (population structure, economic level, etc.). Secondly, the level of expenditure of said neighbours has a significant and positive impact on most subprogrammes, showing the presence of the some of the spatial effects described in Sect. 2 (cooperation, yardstick effects, 
etc.), although in this model is unable to identify the mechanism that generates such dependence. Lastly, and for five subprogrammes, an error spatial structure is identified in the model. From our perspective, the cause that could generate a negative sign in the parameter of spatial dependence in the residuals is related to municipal political decisions, which cannot be included in econometric modelling. Note that this kind of spatial dependence is attributed to the omission of relevant factors that have not been taken into consideration. This evidence would confirm the hypothesis of Lundberg (2006), showing the presence of substitutive mechanisms (which would generate a negative sign) in municipal spending decisions. New research could advance in identifying the causes of these observed effects on local government public spending policies.

Finally, we have to admit that the approach used in the paper may have at least three fundamental weaknesses that merit some further comments. Firstly, it can be argued that the SUR model is static. Although consider a SUR model in a spatiotemporal framework, the temporal dynamics are limited. We do not consider dynamic behaviour due to the short period of analysis, but new results must be obtained when more information is available. Secondly, we do not consider co-dependence between subprogrammes based on the theoretical absence of a causal relationship in the expenditure of different subprogrammes but, as Allers and Elhorst (2011) point out, due to the effective budget restriction in all municipalities, there is economic-theoretical argument to regress one dependent variable on the others ${ }^{4}$ because spending in a subprogramme is linked to spending in the rest of subprogrammes. But in this paper, the expenditure of 10 subprogrammes is around $68 \%$ of the total budget, so the level of co-dependence is probably not very high. Lastly, we consider three types of spatial dependence for each subprogramme (in total $12 * 10+10+10=140$ parameters related to spatial effects are estimated), and therefore, the model could be affected by parameter identification problems (Burridge et al. 2016). Burridge et al. (2016) noted that the model with a full set of interaction effect is almost never used in empirical applications due to two popular misconceptions. The first reason is that to date, no one has proved generals conditions under which the parameters of GNS are identified (except Lee et al. 2010). The second one is that the GNS model can be overparameterized, as result of which the significance levels of all effects tend to fall (Elhorst 2014 p. 33), as a results of which this model does not outperform the more simple SDM or SDE models. But this is not the case for the empirical evidence shown in this paper, where all spatial effects are significant in the SUR-GNS model. In any case, there is scarce literature on this topic and more research is required.

Acknowledgments We gratefully acknowledge the contribution of three anonymous reviewers in helping us improve the quality of this paper. Prof. Fernando A. López, grateful for the financial support offered by the projects from Programa de Ayudas a Grupos de Excelencia de la Región de Murcia, Fundación Seneca (\#19884-GERM-15) and Ministry of Economy and Competiveness (ECO2015-651758-P).

\footnotetext{
4 Thanks to the review for this suggestion
} 


\section{References}

Akai N, Suhara M (2013) Strategic interaction among local governments in Japan: an application to cultural expenditure. Jpn Econ Rev 64:232-247

Allers MA, Elhorst JP (2005) Tax mimicking and yardstick competition among local governments in the Netherlands. Int Tax Public Finance 12(4):493-513

Allers MA, Elhorst JP (2011) A simultaneous equations model of fiscal policy interactions. J Reg Sci 51(2):271-291

Anselin L (1988) Spatial econometrics: methods and models. Kluwer, Dordrecht

Baicker K (2005) The spillover effects of state spending. J Public Econ 89(2):529-544

Baltagi BH, Deng Y (2015) EC3SLS estimator for a simultaneous system of spatial autoregressive equations with random effects. Econom Rev 34(6-10):659-694

Bastida F, Guillamón MD, Benito B (2013) Municipal spending in Spain: spatial approach. J Urban Plan Dev 139(2):79-93

Benito B, Bastida F, Vicente C (2013) Municipal elections and cultural expenditure. J Cult Econ 37:3-32

Brett C, Pinkse J (2000) The determinants of municipal tax rates in British Columbia. Can J Econ 33:695714

Brueckner J (2003) Strategic interaction among governments: an overview of empirical studies. Int Reg Sci Rev 26(2):175-188

Burridge P, Elhorst JP, Zigova K (2016) Group interaction in research and the use of general nesting spatial models. In: LeSage JP, Pace K, Baltagi B (eds) Advances in econometrics, vol 37. Elsevier, Amsterdam

Case A, James H, Harvey R (1993) Budget spillovers and fiscal policy inter- dependence: evidence from the states. J Public Econ 52(3):285-307

Choumert J, Cormier L (2011) The provision of urban parks: an empirical test of spatial spillovers in an urban area using geographic information systems. Ann Reg Sci 47:437-450

Costa H, Veiga LG, Portela M (2015) Interactions in local governments' spending decisions: evidence from Portugal. Reg Stud 49(9):1441-1456

Deng H, Zheng X, Huang N, Li F (2012) Strategic interaction in spending on environmental protection: spatial evidence from Chinese cities. China World Econ 20(5):103-120

Elhorst J (2014) Spatial econometrics: from cross-sectional data to spatial panels. Springer, Berlin

Ermini B, Santolini R (2010) Local expenditure interaction in italian municipalities: Do local council partnerships make a difference? Local Gov Stud 36:655-677

Florax RJ, Folmer H, Rey SJ (2003) Specification searches in spatial econometrics: the relevance of Hendry's methodology. Reg Sci Urban Econ 33(5):557-579

Gebremariam GH, Gebremedhin TG, Schaeffer PV (2012) County-level determinants of local public services in Appalachia: a multivariate spatial autoregressive model approach. Ann Reg Sci 49(1):175-190

Getzner M (2004) Exploring voter preferences in cultural policy: a case study for Austria. Empírica 31:27-42

Griffith DA (2006) Hidden negative spatial autocorrelation. J Geogr Syst 8(4):335-355

Griffith DA, Arbia G (2010) Detecting negative spatial autocorrelation in georeferenced random variables. Int J Geogr Inf Sci 24(3):417-437

Hanes N (2002) Spatial spillover effects in the Swedish local rescue services. Reg Stud 36(5):531-539

Hibbs D (1977) Political parties and macro-economic policy. Am Polit Sci Rev 71(4):1467-1487

Kelejian H, Robinson D (1993) A suggested method of estimation for spatial interdependent models with autocorrelated errors and an application to a county expenditure model. Pap Reg Sci 72:297-312

Lee LF, Liu X, Lin X (2010) Specification and estimation of social interaction models with network structures. Econom J 13(2):145-176

LeSage JP, Pace RK (2009) Introduction to spatial econometrics. CRC Press, Taylor \& FrancisGroup, Boca Raton

Lewis G, Rushton M (2007) Understanding state spending on the arts, 1976-99. State Local Gov Rev 39(2):107-114

López F, Mur J, Angulo A (2014) Spatial model selection strategies in a SUR framework: the case of regional productivity in EU. Ann Reg Sci 53(1):197-220

Lundberg J (2006) Spatial interaction model of spillovers from locally provided public services. Reg Stud 40:631-644

Mur J, Angulo A (2009) Model selection strategies in a spatial setting: some additional results. Reg Sci Urban Econ 39(2):200-213 
Reifschneider A (2006) Competition in the provision of local public goods. Single function jurisdictions and individual choice. Cheltenham, Northampton: Edward Elgar

Revelli F (2006) Performance rating and yardstick competition in social service provision. J Public Econ 90(3):459-475

Revelli F (2001) Spatial patterns in local taxation: tax mimicking or error mimicking? Appl Econ 33(9):1101-1107

Schaltegger C, Zemp S (2003) Spatial spillovers in metropolitan areas: evidence from swiss comunes. Crema 6:1-26

Schulze GG, Rose A (1998) Public orchestra funding in Germany: An empirical investigation. J Cul Econ 22:227-247

Solé-Ollé A (2006) Expenditure spillovers and fiscal interactions: empirical evidence from local governments in Spain. J Urban Econ 59:32-53

St'astná L (2009) Spatial interdependence of local public expenditures: selected evidence from the Czech Republic. Czech Econ Rev 3:7-25

Werck K, Heyndels B, Geys B (2008) The impact of 'central places' on spatial spending patterns: evidence from Flemish local government cultural expenditures. J Cult Econ 58:32-35 